\title{
Building digital life stories for memory support
}

\section{Basel Kikhia* and Josef Hallberg}

Luleå University of Technology, SE-971 87 Luleå, Sweden

E-mail: basel.kikhia@ltu.se

E-mail: josef.hallberg@1tu.se

*Corresponding author

\section{Johan E. Bengtsson}

InterNIT, Parkvägen 2, SE-972 53 Luleå, Sweden

E-mail: johan@internit.se

\section{Stefan Sävenstedt and Kåre Synnes}

Luleå University of Technology, SE-971 87 Luleå, Sweden

E-mail: stefan.savenstedt@1tu.se

E-mail: kare.synnes@1tu.se

\begin{abstract}
The number of persons suffering from dementia is increasing, and there is significant human and economic value to gain by enabling them to keep living independently in their homes. The top priority unmet need is for memory support. This paper introduces context-awareness and life-logging in a system using reminiscence therapy methods, embodied as an ICT memory aid for recording past, current and future activities, which can later be recalled. The tool may help build or maintain episodic memories and self-image, although evidence in this area is lacking. It is designed to also give direct and instrumental support in other priority needs areas. A prototype design is described for a system that is by necessity extremely easy to use, with a touch screen computer in the home and mobile devices for data capture and cognitive support. The main life-log entities associated with the logged activities are places, persons, personal items, and recorded media. Privacy, trust and dignity are key ethical issues.
\end{abstract}

Keywords: digital life stories; memory support; assistive technology; life logging; context awareness; reminiscence methods; dementia.

Reference to this paper should be made as follows: Kikhia, B., Hallberg, J., Bengtsson, J.E., Sävenstedt, S. and Synnes, K. (2010) 'Building digital life stories for memory support', Int. J. Computers in Healthcare, Vol. 1, No. 2, pp.161-176.

Biographical notes: Basel Kikhia received his Bachelor in Informatics Engineering from Tishreen University in 2003. He came to Sweden in 2006 for his Master studies, and graduated with a Master in Information Systems at Luleå University of Technology in 2008. He has worked in CogKnow, a European project for assisting people with mild dementia. He started as a $\mathrm{PhD}$ 
student in 2009 to do research in the MemoryLane Project, which aims at providing a life-log for reminiscence purposes for people with mild dementia. His current research interests are life-logging, context-awareness, and activity recognition.

Josef Hallberg is an Assistant Professor in Media Technology at Luleå University of Technology and is a Key Rresearcher in the Media Technology Research Group within the Department of Computer Science and Electrical Engineering. He has experience working in several EU-level projects, such as MobiHealth and CogKnow. His main research interests lie within ubiquitous and pervasive computing, in context-aware smart environments, and in social networking using cloud computing in particular. He has also published more than 25 academic publications in these areas.

Johan E. Bengtsson is a Certificated Senior Project Manager with 17 years of $R \& D$ experience in internet services, software technology and telecommunications. He has extensive experience in international project management and R\&D strategy work. He had editor, advisor and coordinator roles in the European projects FP7 PROTAGE, FP6 COGKNOW and Esprit ROXY. He has seven years background as a private entrepreneur in system development and education, and further experience as Project Leader, Manager, Project Area Manager and Strategic Advisor, representing TeliaSonera in European steering groups. Since 2004, he is a Private Entrepreneur and Senior Project Manager at Centre for Distance-spanning Technology (CDT) and Centre for Distance-spanning Healthcare (CDH), at Luleå University of Technology. His current research interests are user, technical and business success factors of integrated assistive tools for cognitively disabled. He holds three patents and has published more than 14 papers in refereed journals, magazines and international conferences.

Stefan Sävenstedt is an Assistant Professor in Nursing Care at the Department of Health Sciences, Luleå University of Technology. He is one of the Key Researchers in a research group focusing on research on e-health applications in elder care. The ten years research experience in this field covers many national and international projects and one of the resent projects was the EU-COGKNOW project. His present research interest lies in e-health applications to support persons with dementia.

Kåre Synnes, an Assistant Professor in Media Technology, is doing research in pervasive computing and multimedia communication. He is an Affiliated Researcher at the Centre for Distance-spanning Technology (CDT) and is a member of the scientific board for the Centre for Distance-spanning Healthcare $(\mathrm{CDH}) . \mathrm{He}$ is also the Head of the Division of Information and Communication Technology (ICT) at the Department of Computer Science and Electrical Engineering (CSEE). He is also the Coordinator of studies in media technology within the program Arena Media, Music and Technology. Since 1995, he has been involved in European projects such as FP4 MATES, FP5 Roxy, FP5 MediaSite, FP5 MobiHealth, FP6 CogKnow and FP6 Laboranova. He has also acted as an Expert/Reviewer for European projects since 2009. He is also a Co-founder of the IST Prize winner Marratech AB, a direct result of the FP4 MATES project, which was acquired by Google in May 2007. 


\section{Introduction}

The population suffering from chronic diseases, such as mild dementia, is increasing in our aging society (Pollack, 2005). These persons have difficulties with their daily life as they have problems with remembering names, faces, details of their day, and how to navigate home or to other places. They can also be unable to do even mundane tasks such as preparing a meal, because they cannot remember the steps involved, and if they succeed they may forget to serve it (Rivera-Illingworth et al., 2005). Their ability to maintain an independent living is thus reduced and they risk losing social contacts while becoming increasingly frustrated, affecting not only themselves but also the people around them.

Persons with dementia often rely on external memory aids to help them compensate for their memory deficits (Clare, 2002), such as calendars, diaries, alarms, whiteboards, notebooks, and timers. Those devices have been shown to help persons with dementia maintaining an account of their daily life (Davies et al., 2009; Kapur et al., 2002; Lauriks et al., 2007; Nugent et al., 2007; Roest et al., 2007). The external memory aid builds on the concept of life story work (Lai et al., 2004) where reminiscence provides a sense of control over the past, present and future (Housden, 2007).

This article presents the idea of providing an external memory aid which supports a person with mild dementia in coping with memory problems (Lindeman, 1926) by using context-aware life-logging techniques. Life-logging is recording activities that a person experiences for later retrieval, while context-awareness is reacting on changes in contexts. For example, logging a picture when a person changes location. The aim is to create a semi-automated system which helps persons with mild dementia in supporting and maintaining their life story.

The proposed system enables real-time support and media-enriched life-logs for integrated assistive services by utilising established reminiscence processes and state-of-the-art technology. There are a number of challenges associated with this type of system. The weight and size of worn devices needs to be kept to a minimum, which affects battery life and utilisation of sensors thus limiting capture of life-log entities. Furthermore, the system needs to be easy to operate while addressing privacy carefully. The service for reviewing and reminiscing must also be highly intuitive even if a carer will assist in the review process by annotating names and further details.

In summary, the challenges considered in this article are:

1 Which data and life-log entities are realistic and useful to capture to support reminiscence for a person with mild dementia?

2 How can reviewing and reminiscing be simplified for a person with mild dementia?

The rest of this article is organised as follows. The next section reviews the state-of-the-art in reminiscence and life story work. Section 3 describes a scenario which presents the functionality of the proposed system. Section 4 describes the concepts behind creating a life story using context and content. The architecture of the target system is presented in Section 5, and Section 6 discusses ethical issues. Finally, the discussion of the research questions is presented in Section 7 and Section 8 concludes the article by describing future work. 


\section{Reminiscence and life story}

Reminiscence has been described as "dwelling on the past and as retrospection, both purposive and spontaneous" (Havighurst and Glasser, 1972), "the act or process of recalling the past" (Butler, 1963), and "the remembered past" (Lieberman and Falk, 1971). Remembering the past helps any person in re-examining life, recalling past activities and accomplishments, pursuing remote memories, and seeking personal validation. This brings new awareness to the present and a sense of control over their past, present and future (Housden, 2007).

For persons suffering from dementia, memories can be confused and disconnected while accompanied by difficulties of experiencing wholeness and meaning (Kihlgren et al., 1944). Persistence of self across time can be maintained, despite the cognition and memory problems in dementia, through i.e., cognitive socialisation (Bassett and Graham, 2007), especially if done together with others. Reminiscence processes can support persons with dementia to maintain a life story and thereby maintaining the self (Surr, 2006). The reminiscence interventions described in literature varies in content and are often described as using personal photos, visual and verbal prompts, collection of verbal life stories, use of music, movements, and role plays (Moos and Björn, 2006). Reminiscence methods include:

- Reminiscence therapy involves the discussion of past activities and events with other persons, usually with the aid of tangible prompts such as photographs, daily life items, music and archived sound recordings. It is a frequent psychosocial intervention in dementia care which is highly rated by staff and participants (Woods et al., 2005). It also helps in decreasing depression symptoms and in facilitating social involvement for persons (Gibson, 1994).

- Life review is a method for use in reminiscence processes that typically involves individual sessions, in which the participants are guided chronologically through life experiences, encouraged to evaluate them, and possibly to produce a life story book (Woods et al., 2005).

- Life story work is also a method used in reminiscence processes, which is a biographical approach in health and social care that gives persons the opportunity to talk about their life experiences (McKeown et al., 2006).

The overarching aims of life story work are to help uncover or preserve the identity or personhood of the person with dementia, improve quality of life through the impact of being listened to, and to help staff better understand the person and to improve the provision of care (McKeown et al., 2006). Persons with dementia indicate appreciation for being in focus and valued as a person (Clarke et al., 2003) and that their psychosocial well-being is improved (Lai et al., 2004).

There is, however, no evidence that life story work or other cognitive training methods can improve cognition and behaviour for a person with dementia (Clare and Woods, 2003; Pusey, 2000). A Cochrane review (Woods et al., 2005) indicates positive effects of reminiscence work in general on quality of life and reduced depression, but that a robust conclusion is difficult to make due to the small size and relatively low quality of the few studies done. Many studies on reminiscence interventions focus on persons in advanced stages of dementia and living in nursing home settings, but there are studies 
positively evaluating reminiscence work among persons with mild dementia (Chung, 2009).

\section{Scenario}

This section describes a scenario featuring Lisa who is a woman suffering from mild dementia. A context-aware life-logging system was provided to Lisa to support her. A cognitive assistant, which is an application for accessing previously logged activities, persons, and places, was installed in her mobile device. Furthermore, a review client, which is an application for organising, sorting, and reviewing information that has been logged throughout the day, was installed in a touch screen computer at home. The scenario describes how the system can help Lisa throughout the day, and how reminiscence can be carried out by her together with her husband in order to support and maintain the life story.

The scenario below describes how the cognitive assistant provides Lisa with real-time support, reminds her about her doctor's name, past activities with him, and guides her on the way back home. The scenario also describes the review process where Lisa has the opportunity to recall past activities, and to rehearse important information she may want to remember in the future. In the reviewing process, Lisa is assisted by her husband who helps in naming activities and in adding details which are not automatically detected by the system. Reviewing activities in this way may help in building lasting episodic memories (Bassett and Graham, 2007); it makes it possible to recall past activities at a later time, and to connect fragmented memories from the past with activities, places, and persons in the present which help support the life story.

Finally, the scenario shows that future activities can be added to the system and that the system could fill in significant life-log data and auto-recorded media at the time of the activity. Reminiscence processes are supported within the system through both the cognitive assistant (last activities with a known person, previous visit to a place, etc.) and the review client (rehearsing the day, recalling past activities, etc.). The goal with this technology is to give the person with dementia the tools and information to support maintaining their life story through memories of important events, places, and people that connects past with present.

Lisa is on her way to the medical centre for her medical check-up. Today, she is meeting with Dr. Stefan, and as she sits down in the waiting room she is reminded of his name by the mobile device that has detected the doctor's presence. While she is waiting she takes the opportunity to recall and review past activities where Dr. Stefan has been present. Lisa's automatic camera attached to her chest transparently records images of her surroundings. Dr. Stefan asks Lisa if she is going to turn off the recording of images during the visit. - "Of course Stefan", Lisa says, while pushing the big red button on the camera. When Lisa finishes the medical check-up and leaves, she picks up her mobile device again, and she starts following the navigation instructions given to her by the mobile to find her way home.

At the end of the day, when Lisa is back in her home, she and her husband sit down to review the activities of the day. Collected data from her camera and mobile phone are automatically uploaded to the home computer and they start reviewing a suggested set of activities for the day (some were taken directly from her electronic calendar). Together, 
they select representative images for each of the different activities: a few images from the clinic, an image from the laundry room, and images from the dinner that Lisa had with her friends that day. Lisa watches the screen the entire time and sometimes asks to move back to repeat the names.

Detected persons and places are already indicated by the review client, and Lisa's husband helps her to add information about two of her friends. Once they are done with the review process, Lisa's husband helps her add "Going to the day centre tomorrow 10:00-14:00". They also add 'day centre' as a new place. Even though it would be possible to add the exact location of this place, Lisa's husband decides that it is not necessary, since he will be accompanying her on this first visit, and the system will figure out the exact location during the activity. "I hope the others at the day care centre will not be too curious about Lisa's memory support tool" he thinks. After reviewing the day, Lisa takes the opportunity as well to browse via her sister's page to recall that dinner last week when her sister was there.

\section{Creating the life story}

Tulving (1984) suggested that our memories are stored as 'episodes' which are similar to story form. Life story allows persons to share and communicate their experiences with others, and it also offers them the opportunity for reminiscence and reflection (Byrne and Jones, 2008). Recording the life of a person with mild dementia should be done without creating any burden for the person.

\subsection{Content and context logs}

Collecting data in context-aware life-logging systems to support reminiscence processes require two different logs:

1 Context log: Any information that can be used to characterise the situation of an entity (person, place, or object) (Dey, 2001). Equipment and sensors will record daily activities of the person with mild dementia. Sensors include, but not limited to, an image capturing sensor, Bluetooth detection sensor, and GPS. The data that are captured from those devices will be filtered and aggregated by the system to detect the places that the person visited, the met persons, and the used objects throughout the day.

2 Content log: In addition to the context log, recording daily activities will include content such as images, audio or even video.

It is important to collect enough data to provide sufficient material for reviewing the activities at the end of the day. This may be problematic outside the home since the system has to rely on data collected by equipment carried by the person with mild dementia, while the system can collect data from a sensorised environment inside the home. However, context and content logs have different impact on reminiscence processes and memory recollection, so it is fundamental to collect proper logs of both and use them effectively to support memory recall. 


\subsection{Life-logging entities}

Life-logging entities are memory concepts that capture the life in a detailed way. Information about these entities can be used to provide memory support for a person with mild dementia. An activity entity is a period of time with a specific purpose in daily life (e.g., shopping, going to medical centre). The idea is to actualise episodic memory by visualising explicit links to related entities in the semantic memory of the person with mild dementia.

Known persons and their relations to the person with dementia are significant as this provides contextual links to semantic memory. The place entity provides contextual links to other activities at the same location, and has annotations about the exact location and the significance of the place to the user. The places in a day are also helpful in partitioning the day into distinct activities based on the visited places. Items brought along are also significant entities indicating types of activities and modes of transport.

Each entity can also be associated to recorded media describing them, which might help in training the semantic memory of a person with mild dementia. The authors' hypothesis is thus that the main significant conceptual entities for forming a life-log are places visited, persons met, personal items brought along, activities, and recorded media. Forming life-logs using these life-log entities has been supported by previous studies (Jones et al., 2008; Lee and Dey, 2006) but the effectiveness of these life-log entities for building lasting episodic memory remains to be tested.

Initial feasibility analysis indicates that modern mobile phones are capable of detecting places by using built in GPS (outdoors) or Bluetooth beacons (indoors), while persons and items can be sensed by detecting pre-registered Bluetooth addresses that have been used as tags. However, social practices of having Bluetooth enabled with a name that allows at least limited identification of the person are not in common use, so practical systems may need to promote such practices. Detecting significant new places can be done through social practices of naming stationary Bluetooth devices, or by location estimation through interpolation and hierarchical clustering of GPS data. Prototype recording devices like the Microsoft SenseCam (Sellen et al., 2007) and its upcoming commercial version Vicon Revue can be used for automatic recording of images and some environmental data (Gemmell et al., 2004). There are early results on image-based extraction of context data (McKeown et al., 2006) and locations, although, the processing requirements are still preventing their use to provide real-time advice to users.

\section{Architecture}

A thought-through architecture is needed for the system that will capture the life-log and enable making annotations of significance to the person with mild dementia. The architecture focuses on support for reminiscence and having access to information about previous activities to support memory recollection. The authors combine results from previous context-aware system studies in a tool that can assist a person with mild dementia, indoors and outdoors. The authors extend previous work in life-logging by introducing a review client which use the reminiscence therapy method described in Section 2, and makes it possible to visualise the life-log as sets of non-overlapping activities that are mostly determined by the system. Moreover, a main goal for the target 
system is reducing the need for persons with mild dementia and their carers to manually associate recorded media and context with the activities.

Figure 1 shows the data and operations flow for the intended memory support system. Each step is indicated by a letter to clarify the sequence of the data flow. Sensor data such as Bluetooth and GPS samples, and media such as images, are collected automatically by devices that are carried by the person, or present in the local environment (a). This requires the user to bring the relevant equipment and to have it turned on. The data will then be time-stamped and transferred to local storage in the home station computer (b). Transferring the data is done automatically as soon as the mobile device is connected to the home station (while simultaneously charging the device). When the person with dementia gets back home, an initial wireless transfer of data might occur so that the first unreviewed activity becomes quickly available in the home station. When the battery is running low, or at suitable times like lunch time, the user will be prompted to connect the mobile device to the home station.

Figure 1 The system architecture

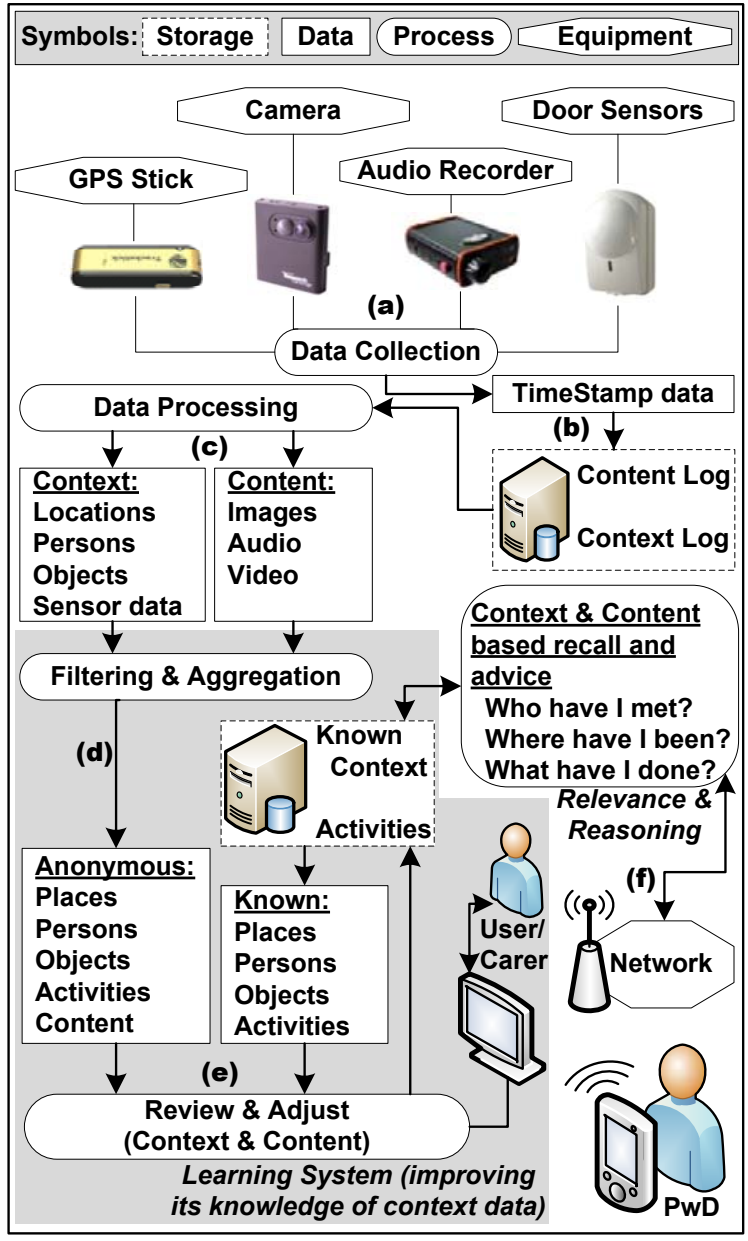


When collected data has been uploaded to the home station, it is processed (c) to detect life-log entities from context data (locations, persons, and items) and to organise the content data into media collections (images, audio, and video). The life-log entities and media collections will then be automatically analysed, filtered and clustered into activities, by time-correlating them (d).

After the data has been clustered into activities, the person with mild dementia, together with the carer, can review and adjust the activities and its associated memory entities and media (e). The carer can assist by selecting media items, adjusting titles and times of activities, naming places (if not already known), and naming persons appearing in the activities (if not already known). At this stage, the person with mild dementia also has the opportunity to recall the activities of the day, for reminiscence purposes or to possibly help build episodic memories. Figure 2 shows a design of the activity review interface. It presents the person with mild dementia and the carer with a view of the activity with a preliminary selection of place, persons and media, based on the collected data. The user can review the activity, choose relevant media, change the representative image, and also specify the place and present persons. The last step in the data flow is to let the person with mild dementia later recall the activities, and receive pro-active advice based on past, current and known future context (f).

Figure 2 The activity review interface

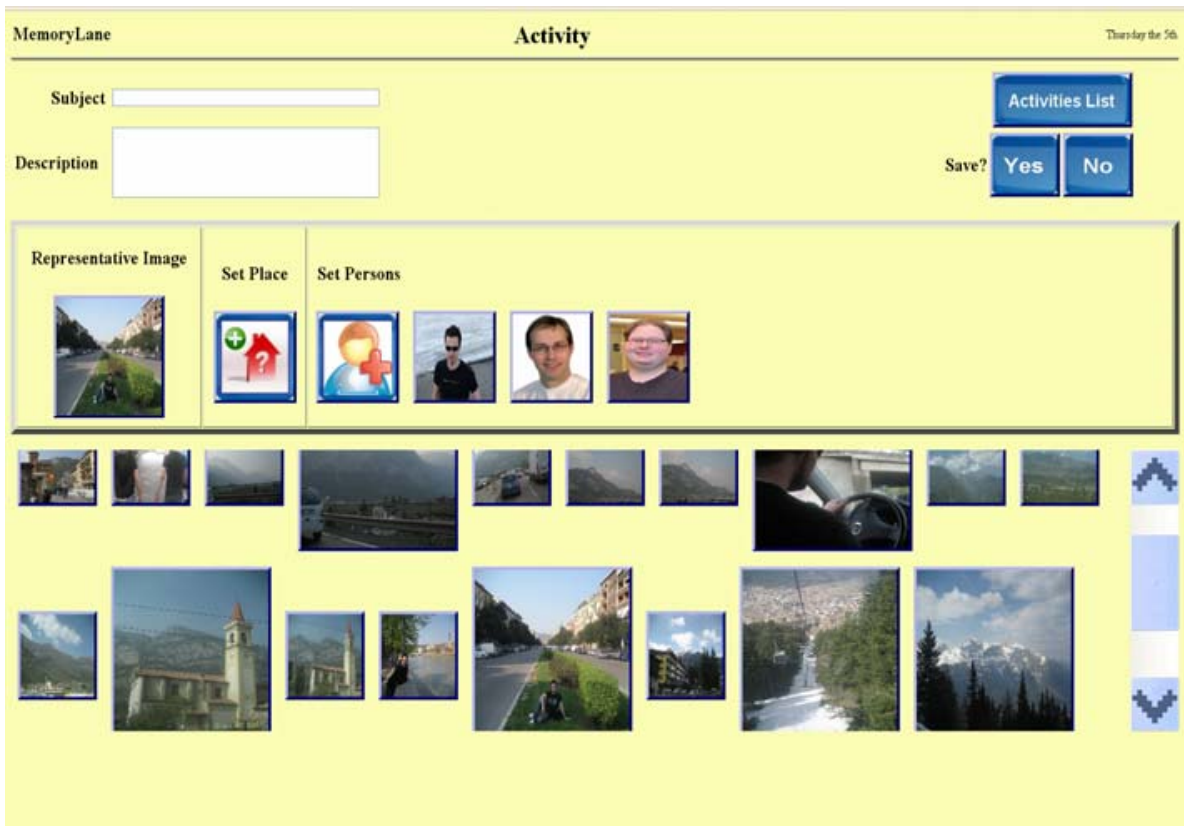

The user interface in the review client is intended to be extremely simple and intuitive to use. It involves very few steps and offers only a few selectable options to make it easier to operate, even for persons who are not very capable computer users. General decline in perception abilities of elderly persons and the prevalence of other disabilities must also be considered, applying universal design principles to create an inclusive interface. 
All context entities are stored and used in future review sessions, and they are also available for use in the mobile device to offer context-based association to past and known future activities. The mobile cognitive assistant is intended to support the person with mild dementia throughout the day. Figure 3 shows the main interface of the mobile cognitive assistant, which includes time indication, nearby people indication, an indication of the next known activity, and an icon to access information about the current place.

Figure 3 The main interface of the mobile cognitive assistant

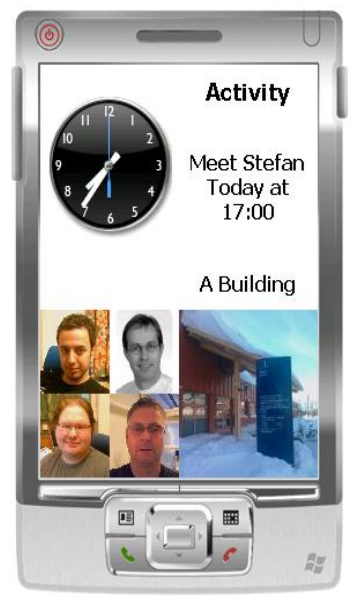

Carefully designing a simple operation flow and ensuring that enough data is collected to automatically identify context entities is essential to enable daily routines of use that are not burdening the person with mild dementia or carers. Persons with mild dementia, often have problems operating advanced technology because of difficulties in remembering and acting, so it is must be determined which tasks can be handled by the person with mild dementia alone, and which tasks should be handled with the assistance of a carer. Also, the cognitive capabilities vary widely between persons and over time. The complexity of the interface and the information presented must be kept low, with consistent use of few and intuitive icons. Multi-modal indications and prompts will help persons with cognitive impairments trigger memories and action, and can compensate for any perception impairments. For example, image icons should be combined with text labels, and prompts should combine text, image and voice whenever these alternatives are available.

With advanced algorithms it is possible to deduce activities from the life-log data. Many techniques have been proposed that can contribute to an effective method in recognising activities, e.g., using context to search parts of recorded video (Aizawa and Hori, 2003), use of a wearable camera for capturing video and a sensor of brain waves (Aizawa et al., 2001), face detection and low level analysis of images (Aizawa and Hori, 2003; Cooray et al., 2006; Doherty and Smeaton, 2008; Spyrou et al., 2005). As a general rule, the more sensor data the system collects, the more accurate it can be in recognising and classifying activities. The system presented in this article will initially detect activities based on: 
a time spent at each location, where the name of the location is assumed to give some information about the activity

b time spent together with known persons (socialising).

However, the authors believe it may be possible to train the system to more accurately detect specific activities that are relevant to the person with mild dementia.

Advanced algorithms can filter out unwanted images and select a small set of likely candidates for images to keep. The aim is to reduce the total amount of images from a day, which could be well over 2000 images, to a small set of perhaps 50 images with good quality. This is achieved in a first step by filtering out black, blurry, and similar images. The clustering of collected data into activities also means that images that do not belong to any activity will not be seen. Another aim is to automate as much of the review process as possible by automatically detecting life-log entities (place, person, personal item), and also to provide a default activity description based on these life-log entities, such as "You were in the park with Johan, then Jim joined you later on".

In addition to automatic capturing, it is essential to support manual adding of past and future activities to the life-story, with associated known place, persons, personal items and relevant media. Captured context and content for the set time period should be automatically added as soon as they are available (for past activities immediately, followed by reviewing the activity). As a special case, the time period of a past activity should be possible to set using the detailed location track in a map view, or by fast-forwarding through all images taken during the day.

\section{Ethical considerations}

Some authors have criticised the idea of intelligent environments and embedding technology in someone's daily life. It has been said that the technology is a "feverish dream of spooks and spies to plant a 'bug' in every object" (Talbott, 2000), and "an attempt at a violent technological penetration of everyday life" (Araya, 1995). A system which automatically captures life-log entities and records the day is bound to capture some sensitive personal information. Such information could include sensitive conversations, sensitive images, and perhaps locations which the user might not wish to disclose. It is important to take necessary measures to limit privacy intrusion. However, balancing privacy and gain is an art, and will be highly individual.

There can sometimes be unwanted side effects when using intelligent environments to compensate for cognitive impairments. The technology may, i.e., place the user in an unfamiliar situation which can be difficult to handle. For example, navigation tools may, if not properly adjusted, take the user into an unfamiliar environment. However, despite the risks the potential benefit of such functionality motivates a further evaluation of what benefits it can provide for persons with mild dementia.

One very natural privacy function is the ability to turn everything off. However, it can be easy to forget to turn everything back on, so a function for turning things off for a short time is also helpful. This function is useful for when, e.g., going to the bathroom, while it may be more useful to turn everything off completely when attending longer meetings which should not be recorded. Context-triggered turn-offs will also be useful. 
Different media are more sensitive than others, which mean, it is important to consider what sensors are being used when recording the day. Location may not be so sensitive, depending on the granularity of the position, but audio can be highly sensitive (Nguyen et al., 2009). Visual imagery may be more important for remembering, however, audio still provide a major benefit during reminiscence (Rubin et al., 2003) though maybe difficult and sensitive to review. The audio may be of poor quality because of background noise, it may have captured conversations which the user was not aware of and which may be sensitive in nature, and audio contains more details of a conversation than, i.e., images. Taking the privacy of others featured on an audio recording into consideration it may therefore be wise not to record audio at all and instead focus on capturing images which can later be annotated by the user together with the carer.

Images are very useful for reminiscence, but these can feature sensitive material as well. As previously mentioned, one way of reducing the number of privacy sensitive images is simply to turn the system off. However, with proper training of image filtering algorithms it is possible to filter out some images with sensitive content, i.e., by matching the lighting in images to the specific lighting conditions in some bathrooms. However, state-of-the-art filtering algorithms are not perfect, but if the image material consists of over 2000 images it may be acceptable to be overambitious in the filtering process. After all, the goal is to limit the number of images which need to be reviewed at the end of the day.

\section{Discussion}

In Section 1, two challenges were listed. The first challenge "Which data and life-log entities are realistic and useful to capture to support reminiscence for a person with mild dementia?" has been addressed by surveying previous work and studies in the area. The result culminates in a system that utilises the memory entities place, person, items, recorded media (e.g., images), and activities which are derived from other entities and user input. These entities can be captured by simple sensors which are readily available in mobile devices.

When creating a life story for a person with mild dementia, the user should participate in giving meaningful names for the life-log entities; however, for some of the entities the system may be able to add such information automatically. For instance, when the collected data are transferred to the system by the end of the day, the system can create narrative threads, such as "You were in the park with Johan, then Jim joined you later on" to describe activities. Associating semantic meaning with the data will simplify the interaction between the person with mild dementia and the system, and it will improve the construction of the life story which will be used in reminiscence processes later on.

The second challenge "How can reviewing and reminiscence of the day be simplified for a person with mild dementia?" has been addressed by describing an architecture of a context-aware life-logging system. The data and operation flow has been designed to be easy to understand and operate for the person with mild dementia - it has to be. The gathering of data only requires the person with mild dementia to carry the data capture devices and make sure they are turned on. The system then aggregates collected life-log entities automatically, and organises them into separate activities. The vast amount of 
image data can be reduced by applying image filtering algorithms which sorts out black, blurry, unwanted, and similar images.

The review client in the target system uses the reminiscence therapy method which was introduced in dementia care over 20 years ago. It is also based on the idea of letting the person with mild dementia review the activities of the day together with a carer. The carer can help in navigating the user interface as well as in filling some information that the person with mild dementia may have forgotten. Together, they can organise and sort the collected activities of the day so that these can be recalled later. The person with mild dementia can also choose to rehearse certain details that may be of particular importance in order to support and maintain the life story. This was indicated to be effective for building durable episodic memories in a study of Basset and Graham (2007).

The previously discussed challenges post some technical and ethical challenges when providing a life-log system for reminiscence. The main technical challenge is in the activity recognition task. "Activity recognition is a key component for creating intelligent, multi-agent systems" (Vail et al., 2007). This recognition should be done automatically so that each day of a person is divided into relevant activity blocks. It may also be useful to divide activities into sub-activities, such as talking to a friend on the phone while waiting for a doctor's appointment.

Ethical challenges when providing a life-log system for reminiscence are of course privacy, trust, and dignity. Neither of these have been the focus of this article, although some privacy considerations have been mentioned. Nevertheless, the authors acknowledge these important issues and propose to integrate existing security solutions into the architecture.

\section{Conclusions and future work}

In this article, we have described our hypothesis on which memory entities are significant for building life-logs. We have also reviewed the evidence that reminiscence processes are relevant to persons with mild dementia, and the viability of creating a semi-automated tool with a simple-to-use interface for carrying out reminiscence tasks. The target system provides real-time services and the possibility to recall past activities and plan future ones. The authors are working on the hypothesis that reviewing and recalling activities, in addition to the real-time services, will support memory of persons with mild dementia and improve the quality of their life.

There are still a number of research areas which need to be addressed, such as creating advanced activity recognition and image filtering. Our challenging aim is now to develop a usable memory support system, and to perform naturalistic evaluation of our hypotheses in field tests with real users. The plan is to teach the users the system through expert assisted tutorials, video tutorials, and manuals. By providing information about activities throughout the day, even mundane and routine activities, we hope to help support the life story. The person with mild dementia, together with a carer, can then decide which information is worth keeping for the future. It is the hope of the authors that this, together with real-time support, will improve the quality of life for persons suffering from mild dementia. 


\section{Acknowledgements}

Our thanks go to our colleagues in the MemoryLane project, http://www.memorylane.nu/. This work is part of the concept and specification phase of the MemoryLane Project. This work has been co-funded by the European Regional Development Funds, Mål-2.

\section{References}

Aizawa, K. and Hori, T. (2003) 'Context-based video retrieval system for the life-log applications', in Proc. of MIR 2003, pp.31-38, ACM, New York.

Aizawa, K., Ishijima, K. and Shiina, M. (2001) 'Summarizing wearable video', Proceedings of the International Conference on Image Processing 2001 (ICIP), IEEE, Vol. 3, pp.398-401.

Araya, A.A. (1995) 'Questioning ubiquitous computing', in Proceedings of the 1995 ACM 23rd Annual Conference on Computer Science, ACM Press, ISBN: 0-89791-737-5.

Bassett, R. and Graham, J. (2007) 'Memorabilities: enduring relationships, memories and abilities in dementia', Aging and Society, Vol. 27, pp.533-554.

Butler, R.N. (1963) 'The life review: an interpretation of reminiscence in the aged', Psychiatry, Vol. 26, pp.65-76.

Byrne, D. and Jones, G. (2008) 'Towards computational autobiographical narratives through human digital memories', 2nd ACM Workshop on Story Representation, Mechanism and Context - SRMC08 at ACM Multimedia 2008, Vancouver, Canada.

Chung, J.C.C. (2009) 'An intergenerational reminiscence program for older adults with early dementia and youth volunteers: values and challenges', Scandinavian Journal of Caring Sciences, Vol. 23, pp.259-264.

Clare, L. (2002) 'We'll fight it as long as we can: coping with the onset of Alzheimer's disease', Aging \& Mental Health 2002, Vol. 6, No. 2, pp.139-148.

Clare, L. and Woods, B. (2003) 'Cognitive rehabilitation and cognitive training for early-stage Alzheimer's disease and vascular dementia', Cochrane Database of Systematic Reviews 2003, No. 4, Art. No.: CD003260, DOI: 10.1002/14651858.CD003260.

Clarke, A., Hanson, E. and Ross, H. (2003) 'Seeing the person behind the patient: enhancing the care of older people using a biographical approach', Journal of clinical Nursing, Vol. 12, No. 5, pp.697-706.

Cooray, S., O’Connor, N.E., Gurrin, C., Jones, G.J.F., O’Hare, N. and Smeaton, A.F. (2006) 'Identifying person re-occurrences for personal photo management applications', in VIE, pp.144-149, Bangalore, India.

Davies, R.J., Nugent, C.D., Donnelly, M., Hettinga, M., Meiland, F., Moelaert, F., Mulvenna, M.D., Bengtsson, J.E., Craig, D. and Droes, R-M. (2009) 'A user-driven approach to develop a cognitive prosthetic to address unmet needs of people with mild dementia', Journal of Pervasive and Mobile Computing, Vol. 5, No. 3, pp.253-267, ISSN: 1574-1192

Dey, A.K. (2001) 'Understanding and using context', Personal Ubiquitous Computing, Vol. 5, No. 1, pp.4-7.

Doherty, A.R. and Smeaton, A.F. (2008) 'Combining face detection and novelty to identify important events in a visual lifeLog', in IEEE International Conference on Computer and Information Technology, Workshop on Image- and Video-based Pattern Analysis and Applications, Sydney, Australia.

Gemmell, J., Williams, L., Wood, K., Lueder, R. and Bell, G. (2004) 'Passive capture and ensuing issues for a personal lifetime store', in 1st ACM Workshop on Continuous Archival and Retrieval of Personal Experiences, ACM, October, pp.48-55. 
Gibson, F. (1994) 'What can reminiscence contribute to people with dementia?', in Bornat, J. (Ed.): Reminiscence Reviewed: Evaluation, Achievements, Perspectives, pp.46-60, Open University Press, Buckingham.

Havighurst, R.J. and Glasser, R. (1972) 'An exploratory study of reminiscence', Journal of Gerontology, Vol. 27, pp.235-253.

Housden, S. (2007) Reminiscence and Lifelong Learning, National Institute of Adult Continuing Education, ISBN: 9781862012486 , Leicester.

Jones, G., Gurrin, C., Kelly, L., Byrne, D. and Chen, Y. (2008) 'Information access tasks and evaluation for personal lifelogs', in Proceedings of the Second International Workshop on Evaluating Information Access (EVIA), December, pp.75-86, Tokyo, Japan.

Kapur, N., Glisky, E.L. and Wilson, B.A. (2002) 'External memory aids and computers in memory rehabilitation', in Baddeley, A.D., Kopelman, M.D. and Wilson, B.A. (Eds.): The Handbook of Memory Disorders, 2nd ed., pp.757-784, John Wiley, Chichester, UK.

Kihlgren, M., Hallgren, A., Norberg, A. and Karlsson, I. (1994) 'Integrity promoting care of demented patients: pattern of interaction during morning care', International Journal of Aging and Human Development, Vol. 39, pp.303-319.

Lai, C.K.Y., Chi, I. and Kayser-Jones, J. (2004) 'A randomized controlled trial of a specific approach to promote the wellbeing of nursing home residents with dementia', International Psycho Geriatrics, Vol. 16, No. 1, pp.33-49.

Lee, M.L. and Dey, A.K. (2006) Capturing and Reviewing Context in Memory Aids, Human-Computer Interaction Institute, Presented in Workshop on Designing Technology for People with Cognitive Impairments, Carnegie Mellon University, Pittsburgh, PA.

Lieberman, M.A. and Falk, J.M. (1971) 'The remembered past as a source of data for research on the life cycle', Human Development, Vol. 14, pp.132-141.

Lindeman, E.C. (1926) The Meaning of Adult Education, New Republic, Redistributed edition 1989, New York.

McKeown, J., Clarke, A. and Repper, J. (2006) 'Life story work in health and social care: systematic literature review', Journal of Advanced Nursing, Vol. 55, No. 2, pp.237-247.

Moos, I. and Björn, A. (2006) 'Use of the life story in institutional care of people with dementia: a review of intervention studies', Aging and Society, Vol. 26, pp.431-454.

Nguyen, D.H., Marcu, G., Hayes, G.R., Truong, K.N., Scott, J., Langheinrich, M. and Roduner, C. (2009) 'Encountering SenseCam: personal recording technologies in everyday life', in the Proceedings of UBICOMP 2009: The 11th International Conference on Ubiquitous Computing, 30 September-3 October, Orlando, FL.

Nugent, C.D., Mulvenna, M.D., Moelaert, F., Bergvall-Kareborn, B., Meiland, F., Craig, D., Davies, R., Reinersmann, A., Hettinga, M., Andersson, A-L., Droes, R-M. and Bengtsson, J. (2007) 'Home-based assistive technologies for people with mild dementia', in Okadome, T., Yamazaki, T. and Mokhtari, M. (Eds.): Pervasive Computing for Quality of Life Enhancement, Lecture Notes in Computer Science, Vol. 4541, pp.63-69, ISBN: 978-3-540-73034-7.

Pollack, M. (2005) 'Intelligent technology for an aging population: the use of ai to assist elders with cognitive impairments', AI Magazine, Vol. 26, No. 2, pp.9-24.

Pusey, M.K. (2000) 'Dementia care: intervention with people with dementia and their informal carers', Mental Health Care, Vol. 3, pp.204-207.

Rivera-Illingworth, F., Callaghan, V. and Hagras, H. (2005) A Neural Network Agent Based Approach to Activity Detection in AmI Environments, 28 June, pp.92-99, ISBN: 0863415180, Colchester, UK.

Roest, H.G., Meiland, F.J.M., Maroccini, R., Comijs, H.C., Jonker, C. and Dröes, R.M. (2007) 'Subjective needs of people with dementia: a review of the literature', Int. Psychogeriatr., Vol. 4, pp.1-34.

Rubin, D.C., Schrauf, R.W. and Greenberg, D.L. (2003) 'Belief and recollection of autobiographical memories', Memory and Cognition, Vol. 31, pp.887-901. 
Sellen, A., Fogg, A., Aitken, M., Hodges, S., Roither, C. and Wood, K. (2007) 'Do life-logging technologies support memory for the past? An experimental study using SenseCam', Proceedings of the SIGCHI conference on Human Factors in Computing Ssystems 2007, pp.81-90, ISBN:978-1-59593-593-9

Spyrou, E., Borgne, H., Mailis, T., Cooke, E., Avrithis, Y. and O'Connor, N. (2005) 'Fusing mpeg-7 visual descriptors for image classification', Proceedings of the International Conference on Artificial Neural Networks, pp.847-852.

Surr, C.A. (2006) 'Preservation of self in people with dementia living in residential care: a sociobiographical approach', Social Science and Medicine, Vol. 62, pp.1720-1730

Talbott, S. (2000) The Trouble with Ubiquitous Technology Pushers, or Why We'd Be Better Off without the MIT Media Lab, NetFuture: Technology and Human Responsibility, ISBN: 1-58113-256-5.

Tulving, E. (1984) Elements of Episodic Memory, Oxford Clarendon Press.

Vail, D.L., Veloso, M.M. and Lafferty, J.D. (2007) Conditional Random Fields for Activity Recognition, ISBN: 978-81-904262-7-5, ACM New York, NY, USA.

Woods, B., Spector, A.E., Jones, C.A., Orrell, M. and Davies, S.P. (2005) 'Reminiscence therapy for dementia', Cochrane Database of Systematic Reviews 2005, Art. No. CD001 120, No. 2, DOI: 10.1002/14651858.CD001120.pub 2. 